\title{
LA IDEA DE LOS PRINCIPIOS FORMALES. EL PRINCIPIO DE PROPORCIONALIDAD EN EL CONTROL DE CONSTITUCIONALIDAD
}

\author{
The Idea of Formal Principles. \\ Proportionality in Constitutional Review
}

Martin BOROWSKI*

\begin{abstract}
Sumario:
I. Introducción I.I. La proliferación del principio de proporcionalidad I. II. Una caracterización del principio de proporcionalidad A. El criterio de proporcionalidad B. La proporcionalidad como un criterio sustancial C. Libertad, obligaciones positivas e igualdad I.III. Objeciones al análisis de proporcionalidad II. La "objeción democrática" a la proporcionalidad en el control de constitucionalidad II.I. La "objeción democrática" al control de constitucionalidad II.II. El compromiso entre la democracia y control de constitucionalidad sin discrecionalidad III. La reconstrucción del principio de proporcionalidad por medio de la teoría de los principios III.I. La teoría de los principios A. Principios B. Reglas III.II. El análisis de la proporcionalidad y la teoría principialista IV. Principios sustanciales y formales IV.I. Principios sustanciales IV.II. Principios formales A. Principios formales en búsqueda de principios B. Críticas V. Un principio formal en la ponderación de principios sustanciales V.I. La competencia para crear una meta a optimizar V.II. La decisión autoritativa de ponderar principios sustanciales como la meta a optimizar del principio formal V.III. Tres escenarios V.IV. Los factores para otorgar peso al principio formal A. Peso abstracto B. La intensidad de la interferencia C. Certeza epistémica D. Características del peso de los principios formales y factores genéricos VI. Conclusión VII. Bibliografía
\end{abstract}

Resumen: en este ensayo presento ideas acerca de cómo la dimensión autoritativa del derecho puede conciliarse con la corrección sustantiva en el control de constitucionalidad en el campo del principio de proporcionalidad. Para ello reconstruiré el principio de proporcionalidad por medio de la teoría principialista y sus elementos y rasgos distintivos para después enfocarme en explicar cómo los principios formales y sustanciales operan de acuerdo con la misma lógica, para así sostener que la ponderación conecta o reconcilia la dimensión formal con la sustantiva. Esto me permite concluir que por medio de los principios formales puede refutarse la objeción democrática al principio de proporcionalidad en el control de constitucionalidad.

Palabras clave: proporcionalidad, principios sustanciales, principios formales, derechos fundamentales, control de constitucionalidad.

\footnotetext{
* Traducción de Alejandro Nava Tovar. Profesor-investigador en el Instituto Nacional de Ciencias Penales (INACIPE) y profesor de Filosofía del derecho en el Posgrado en Derecho de la UNAM. Miembro del Sistema Nacional de Investigadores (SNI 1). ORCID: 00oo-0002-5770-5998. Revisión de Arnulfo Daniel Mateos Durán, doctorando en derecho por la Facultad de Derecho de la Universidad Karl-Ruprecht de Heidelberg, Alemania. Martin Borowski es profesor titular de la cátedra de Derecho público, teoría constitucional y filosofía del derecho de la Facultad de Derecho de la Universidad Karl-Ruprecht de Heidelberg, Alemania. Entre sus obras principales se encuentran Grundrechte als Prinzipien (Los derechos fundamentales como principios) y Die Glaubens- und Gewissensfreiheit des Grundgesetzes (La libertad de creencia y de religión en la ley fundamental), así como autor de un notable número de artículos y compilaciones en diversos idiomas sobre teoría constitucional, derecho público y filosofía del derecho. Recientemente acaba de ser publicada la tercera edición de Grundrechte als Prinzipien (Baden-Baden, Nomos 2018). Correo de contacto: borowski@jurs.uni-heidelberg.de.
} 
Abstract: In this essay, I present ideas about how the authoritative dimension of law can be reconciled with the substantive correction in constitutional review in the field of proportionality. In order to do this I proceed to reconstruct proportionality by means of principles theory and its elements, and then I focus on explaining how formal and substantive principles operate according to the same logic, in order to claim that balancing connects or reconciles the formal dimension with the substantive dimension. This will allow me to conclude that the claim that democratic objection to the principle of proportionality in the field of constitutional review can be refuted by means of formal principles.

Keywords: proportionality, substantive principles, formal principles, constitutional rights, constitutional review.

\section{Introducción}

En este ensayo me gustaría esbozar algunas ideas acerca de cómo la dimensión autoritativa del derecho puede conciliarse con la corrección sustantiva. Este problema surge de manera más destacada, aunque no solamente ahí, en el control de constitucionalidad. El análisis del principio de proporcionalidad adquiere un lugar central en el control de constitucionalidad, lo que quiere decir que la consideración de la autoridad legal de ciertas decisiones tiene que ser reconstruida en el marco de la proporcionalidad.

\section{I.I. La proliferación del principio de proporcionalidad}

En un número cada vez mayor, los constitucionalistas han llegado a apreciar que la doctrina de la proporcionalidad es un factor crucial para evaluar pretensiones jurídicas derivadas de los derechos fundamentales. En la jurisprudencia del Tribunal Europeo de Derechos Humanos ubicada en Estrasburgo, la aplicación de los derechos de la convención implica, como regla, el análisis de proporcionalidad ${ }^{1}$. También, el principio de proporcionalidad se ha vuelto un criterio clave en la jurisprudencia de la Corte Europea de Justicia de Luxemburgo en torno a derechos humanos, y en la actualidad está prevista expresamente en la Carta de los Derechos Fundamentales de la Unión Europea, en el apartado primero, cláusula segunda del artículo 51 de tal carta ${ }^{2}$. Es más, el análisis de proporcionalidad ha demostrado su valía en la reivindicación de derechos constitucionales en muchos sistemas jurídicos nacionales. No obstante, la Ley fundamental de Alemania, la Constitución Alemana de 1949, no contempla la proporcionalidad en alguna disposición legal en particular. El Tribunal Constitucional Federal Alemán desarrolló la estructura de la proporcionalidad mediante decisiones importantes de los años cincuenta ${ }^{3}$, y de este modo

\footnotetext{
${ }^{1}$ Cfr., por ejemplo, MARAUHN, Thilo y MERHOF, Katrin (2013), “Grundrechtseingriff und -schranken”, en DÖRR, Oliver et al, EMRK/GG Konkordanzkommentar, vol. 1, Tubinga, Mohr Siebeck, pp. 366-416, números marginales 43-59; PETERS, Anne y ALTWICKLER, Tilmann (2012), Europäische Menschenrechtskonvention, segunda edición, Múnich, C. H. Beck, p. 31-33; HARRIS, David et al (2009), Law of the European Convention on Human Rights, segunda edición, Oxford, Oxford University Press, pp. 349-59; WHITE, Robin y OVEY, Clare (2010), The European Convention on Human Rights, quinta edición, Oxford, Oxford University Press, pp. 325-32.

2 Cfr., por ejemplo, BOROWSKI, Martin (2007), “Limiting Clauses”, en Legisprudence 1, pp. 210-212; FRENZ, Walter (2009), Handbuch Europarecht, Bd. 4 - Europäische Grundrechte, Berlín y Heidelberg, Springer, pp. 183-210.

${ }^{3}$ Intentos previos de entender la proporcionalidad como requerida por la Ley Fundamental de Alemania incluyen artículos fundamentales de Herbert KRÜGER (KRÜGER, Herbert (1950), "Die Einschränkung von Grundrechten nach dem Grundgesetz", Deutsches Verwaltungsblatt 65: 625-628) y DÜRIG, Günter (1956), "Der Grundrechtssatz von der Menschenwürde”, Archiv des öffentlichen Rechts 81, pp. 117-157. A principios de los sesenta, Peter 
la proporcionalidad se ha vuelto un criterio clave para determinar si una ley establecida por el poder legislativo u otro acto estatal es constitucional desde el punto de vista sustantivo ${ }^{4}$.

Más allá de derechos fundamentales y constitucionales, el análisis de proporcionalidad tiene una función central para promover las libertades de mercado en el derecho de la Unión Europea $^{5}$ y las libertades establecidas en el marco de la Organización Mundial del Comercio ${ }^{6}$. Alec Stone Sweet y Jud Mathews han caracterizado el reciente auge internacional del principio de proporcionalidad, originalmente desarrollado en Alemania, del siguiente modo: "al final de la década de los noventa, virtualmente todo sistema constitucional efectivo en el mundo, con la excepción parcial de los Estados Unidos, ha adoptado los principios fundamentales" del análisis de proporcionalidad7. Mientras los estándares sustanciales del control de constitucionalidad en los Estados Unidos el test de racionalidad, la prueba del interés apremiante del Estado $\mathrm{y}$, en algunos ámbitos, el escrutinio intermedio tienen su propia historia, existe, sin embargo, algo por decir en beneficio de la tesis de que los Estados Unidos también adopta algunos elementos de "ponderación". Este problema, junto con el grado en que estos elementos pueden concebirse como parte propia del principio de proporcionalidad, es actualmente objeto de un intenso debate ${ }^{8}$.

\section{II. Una caracterización del principio de proporcionalidad}

\section{A. El criterio de proporcionalidad}

El principio de proporcionalidad en un sentido amplio abarca tres criterios, a saber, idoneidad, necesidad y proporcionalidad en sentido estricto9. La idoneidad requiere que el Estado

LERCHE asumiría que la proporcionalidad cuenta como un principio general del derecho ampliamente aceptado de derecho fundamental (LERCHE, Peter (1961), Übermaß und Verfassungsrecht, Colonia, Carl Heymann), p. 350. En la jurisprudencia de la Corte Constitucional de Alemania pueden encontrarse, respecto del principio de proporcionalidad, dos líneas de razonamiento. Según la primera, la proporcionalidad es concebida como un componente integral del Principio del Estado de derecho (Rechtsstaatsprinzip), BVerfGE 17, 306 (313-4); 23, 127 (133-4); 27, 1 (8); 30, 250 (263); 35, 382 (400); 38, 348 (368); 49, 24 (58); 61, 126 (134); 69, 1 (35); 76, 256 (359); 80,109 (120); 92, 277 (325). En segundo lugar, la corte sostiene que se sigue "de la naturaleza" (aus dem Wesen) de los derechos fundamentales, BVerfGE 19, 342 (348-9); 61, 126 (134); 65, 1 (44); 76, 1 (50); 77, 308 (334).

${ }^{4}$ Cfr., por ejemplo, STERN, Klaus (2011), "Idee und Elemente eines Systems der Grundrechte", en ISENSEE, Josef y KIRCHHOF, Paul (eds.), Handbuch des Staatsrechts der Bundesrepublik Deutschland, vol. IX, Heidelberg, C.F. Müller, p. 112.

${ }^{5}$ Cfr., por ejemplo, CRAIG, Paul y DE BÚRCA, Gráinne (2011), EU Law-Text, Cases, and Materials, quinta edición, Oxford, Oxford University Press, p. 526; BIEBER, Roland et al (2013), Die Europäische Union, décima edición, Baden-Baden, Nomos, pp. 331-335; BOROWSKI, Martin (2012), "Libertades de Mercado y Derechos Fundamentales en la Unión Europea”, en CAPALDO, Griselda et al, Internacionalización del Derecho Constitucional, Constitucionalización del Derecho Internacional, Buenos Aires, Eudeba, p. 395.

${ }^{6}$ En particular, $c f r$., SWEET, Alec Stone y MATTHEWS, Jud (2008), "Proportionality Balancing and Global Constitutionalism”, Columbia Journal of Transnational Law 47, pp. 153-16o, con referencias posteriores.

7 SWEET, Alec Stone y MATTHEWS, Jud (n. 6), p. 75. En general, sobre el auge internacional de la proporcionalidad, cfr., BEATTY, David M. (2004), The Ultimate Rule of Law, Oxford, Oxford University Press; Stone Sweet and Mathews (n. 6), pp. 112-160; BARAK, Aharon (2012), Proportionality, Cambridge, Cambridge University Press, pp. 181-210.

${ }^{8}$ Cfr., COHEN-ELIYA, Moshe y PORAT, Iddo (2010), "American Balancing and German Proportionality: The Historical Origins”, International Journal of Constitutional Law 8, pp. 276-284; BARAK, Aharon (n. 7), en pp. 206-208; ambos con referencias posteriores.

${ }^{9}$ Cfr., ALEXY, Robert (2002), A Theory of Constitutional Rights (trad. de Julian Rivers), Oxford, Oxford University Press, pp. 66-69; CRAIG, Paul y DE BÚRCA, Gráinne (n. 5), p. 545; COHEN-ELIYA, Moshe y PORAT, Iddo (n. 8), p. 267. 
busque un fin legítimo, y que los medios sean apropiados para alcanzarlo, o, por lo menos, que promuevan tal fin. Los fines ilegítimos están excluidos desde el inicio; éstos carecen de un fundamento que pudiese justificar interferencias en derechos fundamentales. Al aplicar el criterio de necesidad, los medios deben tener el efecto menos restrictivo. Esto quiere decir que no hay medios alternativos que infrinjan en un grado menor en los derechos de los individuos, sino que promuevan al menos el fin así como los medios empleados por el Estado. Finalmente, la proporcionalidad en sentido estricto requiere que la interferencia en los derechos del individuo y la promoción de los fines legítimos de la autoridad sean ponderados. Si la primera pesa más que la segunda, la interferencia cuenta como desproporcional y, así, como inconstitucional.

\section{B. La proporcionalidad como un criterio sustancial}

La proporcionalidad es comúnmente empleada como un criterio sustancial para la justificación de una interferencia en un derecho fundamental, por ejemplo, un derecho convencional, fundamental o constitucional. Al seguir el modelo estándar de la estructura de derechos fundamentales ${ }^{10}$, la evaluación de las pretensiones de los derechos es llevada a cabo en tres pasos. Primero, la conducta del titular de derecho debe encuadrarse dentro del área protegida. Si este es el caso, entonces uno debe preguntarse si una autoridad pública ha interferido con el derecho. Si es así, surge la cuestión en torno a si esta interferencia puede justificarse. Usualmente hay tanto criterios formales como sustanciales de justificación. El criterio formal se refiere a la forma de la interferencia. Por ejemplo, puede requerirse que la interferencia sea -o esté legitimada por- un decreto legislativo. El criterio sustantivo se refiere al contenido sustantivo de la interferencia. Por ejemplo, ¿una interferencia es excesiva porque es desproporcionada? En consecuencia, siempre y cuando un acto estatal no se adecúe al criterio de proporcionalidad, entonces este acto es considerado inconstitucional desde el punto de vista sustantivo. Esto corresponde al hecho de que un juicio de ponderación en el control de constitucionalidad necesariamente requiere principios sustanciales. Regresaré a este punto más adelante.

\section{Libertad, obligaciones positivas e igualdad}

El análisis de proporcionalidad fue originalmente desarrollado en el contexto de evaluar pretensiones provenientes de derechos de libertad. No obstante, fue considerado también apropiado en el contexto de obligaciones positivas del Estado, y no menos importante de la obligación constitucional del legislador de promulgar ciertas leyes ${ }^{11}$, y para analizar exigencias provenientes de derechos de igualdad ${ }^{12}$. Esto quiere decir que la proporcionalidad se ha convertido en un criterio universal para evaluar las pretensiones derivadas de los derechos fundamentales de todo tipo.

\section{I.III. Objeciones al análisis de proporcionalidad}

La proliferación del principio de proporcionalidad en el último par de décadas podría considerarse una historia exitosa, en cuanto establece un estándar universal para el control de consti-

\footnotetext{
${ }^{10}$ Cfr., para este modelo estándar, ALEXY (n. 9), pp. 178-222; BOROWSKI, Martin (2007), Grundrechte als Prinzipien, segunda edición, Baden-Baden, Nomos, pp. 231-238.

${ }^{11}$ BOROWSKI, Martin (n. 10), pp. 293-393.

${ }^{12}$ BOROWSKI, Martin (n. 10), pp. 407-454.
} 
tucionalidad en las democracias liberales. El análisis de proporcionalidad —como se mencionó previamente- está firmemente arraigado en la jurisprudencia de muchas cortes constitucionales o supremas y, más recientemente, registrado en documentos constitucionales en el plano nacional y en convenciones y pactos de derecho internacional. La proporcionalidad no fue desarrollada como una idea académica que fue posteriormente puesta en práctica, sino que emergió en las decisiones de las cortes supremas y fue clarificada por medio de una reconstrucción crítica de éstas. En este sentido, el análisis de proporcionalidad está profundamente engranado en la práctica jurídica moderna global.

Sin duda, no todos están dispuestos a acoger el auge del principio de proporcionalidad. Por el contrario, el análisis de proporcionalidad ha dado lugar a temores y a toda una serie de objeciones. En particular, la ponderación ha sido considerada irracional ${ }^{13}$. No obstante, la objeción de considerar a la ponderación como irracional no ha conseguido ser más convincente al repetirse una y otra vez. Durante los inicios de la teoría principialista, la cual sirve como reconstrucción de los principios jurídicos y su característica forma de aplicación, a saber, la ponderación, Ronald Dworkin explicó con una metáfora el sentido en el cual los principios son vinculantes para los jueces. Dworkin hace referencia a principios jurídicos que los jueces están obligados a descubrir y respetar en la textura abierta de las reglas, con lo cual, en consecuencia, tornan así inadecuado el modelo positivista simple de aplicación del derecho ${ }^{14}$. Una objeción a la fuerza vinculatoria de los principios podría ser que la aplicación requiere arbitrio, y la aplicación de las reglas con su textura abierta también requiere arbitrio. Así, un crítico podría preguntar, ¿¿ónde está la diferencia? Dworkin responde con una metáfora: imaginemos a un sargento al que le han dicho sus superiores que mande a cinco hombres a hacer un patrullaje. Él tiene discreción. No obstante, hay diferentes formas de discreción. Si a él se le ordenó que escoja cinco hombres, él tiene discreción en un sentido fuerte. La discreción en un sentido fuerte está caracterizada por la ausencia de estándares establecidos por la autoridad en cuestión ${ }^{15}$. Esto representa la penumbra en duda en el modelo positivista de derecho más allá del núcleo de la certeza ya no hay criterios para determinar si una regla legal es aplicable o no, lo cual implica afirmar que no hay derecho.

En consecuencia, la decisión del juez representa la creación del derecho. Si los superiores le ordenan al sargento que escoja a los cinco hombres más experimentados entonces tiene discreción en un sentido débil. Esta forma de discreción es aplicada por el hecho de que los estándares por aplicar "no pueden aplicarse mecánicamente, sino que exigen el uso de arbitrio" ${ }^{16}$. Uno puede argumentar cuáles son los hombres más experimentados, pero sólo hasta cierto grado. Por ejemplo, algunos hombres pueden tener seis años como soldados en general, pero no han

\footnotetext{
${ }^{13} \mathrm{Cfr}$., en particular, FORSTHOFF, Ernst (1968), "Zur Situation einer heutigen Verfassungslehre”, en BARION, Hans et al, Epirrhosis. Festgabe für Carl Schmitt, Berlín, Duncker \& Humblot: pp. 209; BÖCKENFÖRDE, ErnstWolfgang (1974), "Grundrechtstheorie und Grundrechtsinterpretation", Neue juristische Wochenschrift 27, p. 1534; BÖCKENFÖRDE, Ernst-Wolfgang (2003), "Schutzbereich, Eingriff, verfas-sungsimmanente Schranken. Zur Kritik gegenwärtiger Grundrechtsdogmatik", Der Staat 42, p. 190; HA-BERMAS, Jürgen (1996), Between Facts and Norms, trad. de William Rehg, Cambridge, Polity Press, pp. 253-261; SCHLINK, Bernhard (1976), Abwägung im Verfassungsrecht, Berlín, Duncker \& Humblot, pp. 134 y sigs; SCHLINK, Bernhard (1992), “Grundrechte als Prinzipien?”, Osaka University Law Review 39, p. 55; LEISNER, Walter (1997), Der Abwägungsstaat, Berlín, Duncker \& Humblot, pp. 43 y sigs; JE-STAEDT, Matthias (1999), Grundrechtsentfaltung im Gesetz, Tubinga, Mohr Siebeck, p. 53; MÖLLER, Kai (2007), "Balancing and the Structure of Constitutional Rights", International Constitutional Law 5, pp. 459-461.

${ }^{14}$ DWORKIN, Ronald (1977), Taking Rights Seriously, Cambridge, Harvard University Press, pp. 22 y sigs.

${ }^{15} \mathrm{Ibid}$., en p. 32.

${ }^{16}$ Ibid., p. 31.
} 
estado todavía en combate, mientras que otros pueden tener sólo un año de experiencia como soldados, pero han estado en combate por tres meses. Uno puede argumentar cuáles de ellos son los más experimentados. Sin embargo, es claro que el sargento no obedece su orden si escoge a cinco novatos que llegaron ayer. Esto quiere decir que la discreción en sentido débil es, ceteris paribus, más restringida que la discreción en sentido fuerte. Al tomar esto en cuenta para los principios, estos dejan algo de discreción a la autoridad que está facultada para llevar a cabo la ponderación, no obstante, restringen la discrecionalidad en la aplicación de reglas jurídicas. El moderno análisis estructural de los principios por ponderar ha reconstruido cómo y hasta qué punto esta reducción de la discrecionalidad es efectuada y qué premisas necesitan justificarse en este proceso $^{17}$.

A pesar de ello, debe admitirse que la justificación de los juicios de ponderación en la práctica jurídica es menos que convincente. Por ejemplo, el famoso artículo de Alexander Aleinikoff sobre la ponderación en el derecho constitucional en los Estados Unidos es con frecuencia leído como una crítica respecto a la ponderación. Sin duda, él es cautelosamente optimista respecto a la ponderación como método racional. No obstante, es así porque está a la espera de muchas justificaciones de juicios de ponderación de la corte suprema: "[E]n gran medida, la ponderación tiene lugar en una caja negra"18. Aleinikoff continúa un par de páginas después: "[L]os problemas que aquejan a la mayoría de las opiniones sobre la ponderación, considero, le han provocado un daño severo a la credibilidad de la metodología”"19. Uno tiene que estar en desacuerdo con la conclusión de Aleinikoff. Que un método no sea bien practicado no cuenta en contra del método mismo. El problema que aqueja a muchas opiniones sobre la ponderación, en palabras de Aleinikoff, es la falta de justificación de los pesos atribuidos a los derechos y bienes ponderados en las circunstancias del caso a resolver. Por ejemplo, la Corte Europea de Justicia de Luxemburgo es conocida por sus juicios cortos, los cuales no revelan en buena medida la justificación de las disposiciones operativas de sus argumentos. Esto puede resolverse al desarrollar y aplicar una teoría viable de la argumentación jurídica que se amplíe a la justificación del peso atribuido a los derechos y bienes por ponderar. La ponderación puede estar sujeta a procesos deliberativos en varios niveles: por el público, el gobierno, los comentaristas académicos y por cortes en decisiones subsecuentes si una justificación más detallada requiere ofrecerse.

Sin duda, la aceptación de Dworkin de que la aplicación de principios requiere "discrecionalidad" implica que hay casos difíciles en los cuales personas racionales pueden tener un desacuerdo respecto al resultado de un caso concreto, aun si hay un acuerdo en cuanto a la teoría de la argumentación jurídica a emplear. Esto lleva a la distinción entre escepticismo radical y optimismo moderado en torno a la ponderación. De acuerdo con el escepticismo radical, ningún juicio sobre ponderación puede justificarse con un argumento racional, lo cual es una tesis fuerte. Al asumir, por ejemplo, que la pena capital es impuesta por traspasar el jardín de otro, podríamos afirmar, al margen de las circunstancias, que el derecho a la vida pesa más que el interés de otro en el fomento a preservar su césped. No afirmaríamos que tal juicio es una cuestión de prejuicios y de opinión personal, sino un juicio objetivamente fundamentado. Mucho más razonable es el escepticismo moderado, el cual también puede llamarse optimismo moderado en lo que concierne a la ponderación. De acuerdo con esta posición, la ponderación no lleva de

\footnotetext{
${ }^{17}$ Cfr., ALEXY, Robert (2007), “The Weight Formula”, en STELMACH, Jerzy et al, Studies in the Philoso-phy of Law. Frontiers of the Economic Analysis of Law, Cracovia, Jagiellonian University Press, pp. 9-27; BOROWSKI, Martin (n. 10), pp. 120 y sigs.

${ }^{18}$ ALEINIKOFF, Thomas Alexander (1986/1987), “Constitutional Law in the Age of Balancing”, Yale Law Journal 96, p. 976.

${ }^{19}$ Ibid., p. 982.

CIENCIA JURÍdiCA. Departamento de Derecho. División de Derecho, Política y Gobierno, Universidad de Guanajuato - Año 8, No. 16, 2019
} 
manera racional a un resultado en todo caso concreto, sino sólo en algunos casos, y, de acuerdo con Alexy, lo hace en un número de casos en los que el método de ponderación es de importancia práctica ${ }^{20}$. El optimismo moderado en torno a la ponderación también se percibe en el hecho de que hay muchos casos manifiestos de ponderación, casos en los cuales el resultado de la ponderación es claro. El debate sobre la racionalidad de la ponderación con frecuencia se centra en casos difíciles, los cuales distorsionan la perspectiva en buena medida.

Con seguridad, un análisis detallado de la proporcionalidad y la ponderación, incluyendo las diversas objeciones que han sido ofrecidas, va más allá de los objetivos de este ensayo. Aquí quisiera centrarme en una objeción que se dirige al corazón del análisis de proporcionalidad en tanto es practicado en muchas cortes, a saber, la objeción de que la proporcionalidad no puede servir como fundamento del control de constitucionalidad, ya que la autoridad de las decisiones democráticamente legitimadas no pueden ser reconstruida mediante el análisis de proporcionalidad. Si esto fuera cierto, tendría consecuencias de mucho mayor alcance, esto es, tendríamos que abolir el principio de proporcionalidad en el control de constitucionalidad. No obstante, mi ensayo constituye parte de un esfuerzo por mostrar que esta objeción puede y tiene que ser rechazada. Me encargaré de delinear un modelo que es, espero, a la vez preciso en términos de teoría jurídica e intuitivamente viable en lo que respecta a constatar su razonabilidad.

\section{La "objeción democrática” a la proporcionalidad en el control de constitucionalidad}

Ernst Wolfgang Böckenförde, quien fue juez del Tribunal Constitucional Alemán doce años durante las décadas de los ochenta y noventa, advirtió al final de su mandato que el análisis de proporcionalidad daría lugar a una degeneración de la democracia liberal con un intenso debate en el Estado, en el cual los tribunales de justicia deciden todas las cuestiones importantes por medio del control de constitucionalidad (verfassungsgerichtlicher Jurisdiktionsstaat) ${ }^{21}$. Metafóricamente hablando, esta es la advertencia de que los jueces supremos se convertirían en "jueces reyes" ${ }^{22}$, ya que ellos usurparían la última palabra en cuestiones de legislación. Un buen número de académicos han encontrado esta preocupación verosímil y, por tanto, ha ganado atención considerable en el debate sobre la proporcionalidad dentro y fuera de Alemania.

\section{II.I. La "objeción democrática" al control de constitucionalidad}

De entrada merece señalarse que el control de constitucionalidad en sí mismo se enfrenta a la objeción democrática ${ }^{23}$, por lo que esta objeción al análisis de proporcionalidad en el control de constitucionalidad se vuelve un corolario de la objeción previa. En primer lugar, esto quiere decir que la proporcionalidad está expuesta a la objeción democrática sólo cuando es empleada en el control de constitucionalidad de decisiones tomadas por órganos estatales democráticamente

\footnotetext{
${ }^{20}$ ALEXY, Robert (2002), "Postscript”, en: Robert Alexy, A Theory of Constitutional Rights (trad. De Julian Rivers), Oxford, Oxford University Press, p. 402; ALEXY, Robert (2003), “Die Gewichtsformel”, en JICKELY, Joachim et al, Gedächtnisschrift für Jürgen Sonnenschein, Berlín, Duncker \& Humblot, p. 773.

${ }^{21}$ BÖCKENFÖRDE, Ernst-Wolfgang (1992), “Grundrechte als Grundsatznormen”, en BÖCKENFÖRDE, ErnstWolfgang, Staat, Verfassung, Demokratie, segunda edición, Fráncfort del Meno, Suhrkamp, p. 190.

${ }^{22}$ Ibid

${ }^{23}$ Sobre el debate entre el control de constitucionalidad y la democracia, ver, por ejemplo, HABERMAS, Jürgen (n. 13), pp. 241 y sigs; TUSHNET, Mark (200o), Taking the Constitution away from the Courts, Princeton, Princeton University Press; WALDRON, Jeremy (2006), "The Core of the Case against Judicial Review", Yale Law Journal 115, pp. 1346-1406.
} 
legitimados. Ciertamente esta es una aplicación crucial del principio de proporcionalidad. No obstante, no es su única aplicación. Por ejemplo, si el legislador mismo usa el análisis de proporcionalidad como guía para llevar a cabo un determinado proyecto de ley en el proceso de legislación, entonces no hay nada de antidemocrático en ello. Sólo cuando la proporcionalidad es usada en una corte en el control de constitucionalidad de leyes parlamentarias, la decisión de la corte obtenida por el análisis del principio de proporcionalidad puede interferir con el resultado del proceso democrático, esto es, una ley parlamentaria.

En segundo lugar, el control de constitucionalidad no trata solamente de ponderación. Éste se extiende al análisis de las pretensiones provenientes de las disposiciones constitucionales sobre el todo, y hay elementos de la aplicación de disposiciones de la constitución más allá de la ponderación, a saber, la subsunción ${ }^{24}$. Por ello, surge el problema de la discrecionalidad en la subsunción por razones democráticas. Un modelo amplio de discrecionalidad en el control de constitucionalidad también tiene que abordar esta cuestión ${ }^{25}$. No obstante, me basaré en la discrecionalidad en la ponderación.

En tercer lugar, el problema democrático del control de constitucionalidad es una cuestión que en sí misma ha sido objeto de un prolongado debate ${ }^{26}$. La reconstrucción de la discrecionalidad en la ponderación por medio de los principios formales que será desarrollada a continuación ofrece, creo, la solución a una parte importante del problema democrático en el control de constitucionalidad. En este sentido contribuye a la solución de este problema.

\section{II.II. El compromiso entre la democracia y control de constitucionalidad sin discrecionalidad}

La democracia, tomada en sí misma, no requiere que se realice el control de constitucionalidad de las disposiciones legislativas. El compromiso efectivo de todos los órganos del Estado, entre ellos el legislador parlamentario, a lo que exige la constitución, aparte del principio democrático, requiere que el control estricto de constitucionalidad, el control sin discrecionalidad del parlamento, sea llevado a cabo. No cabe duda de que se está pidiendo un acuerdo razonable entre estos dos requisitos en competencia ${ }^{27}$. Sin duda, la idea de que un parlamento legitimado democráticamente tiene discrecionalidad o de que sus decisiones tienen autoridad, de modo tal que la corte que lleva a cabo el control de constitucionalidad debe respetar, no es nueva. Esta idea puede encontrarse en la jurisprudencia de numerosas cortes nacionales e internacionales. No obstante, "respeto por autoridad" es una metáfora más que un fenómeno legal propiamente reconstruido. Lo que se necesita es un modelo adecuado que explique las características de esta discrecionalidad o autoridad, esto es, ¿cómo encaja éste en el análisis de las pretensiones derivadas de derechos fundamentales, y cómo podemos determinar el grado de la discrecionalidad o el peso de la autoridad de una decisión en el control de la decisión?

Sería un interesante ejercicio en el derecho comparado examinar cómo las diferentes jurisdicciones en el ámbito nacional e internacional abordan este asunto. Debido a diferencias en la tradición jurídica, la cultura jurídica y los diferentes marcos institucionales, probablemente

\footnotetext{
${ }^{24}$ Sobre la reconstrucción de la subsunción, $c f r$., ALEXY, Robert (2003), “On Balancing and Subsumption”, Ratio Juris 16, pp. 433-435.

${ }^{25}$ BOROWSKI, Martin (2009), "Die Bindung an Festsetzungen des Gesetzgebers in der grundrechtlichen Abwägung", en CLÉRICO, Laura y SIECKMANN, Jan-Reinard (eds), Grundrechte, Prinzipien und Argu-mentation, Baden-Baden, Nomos, p. 117.

${ }^{26} \mathrm{Cfr}$., nota 27 .

${ }^{27}$ Cfr., BOROWSKI, Martin (2013), "Formelle Prinzipien und Gewichtsformel”, en KLATT, Matthias (ed), Prinzipientheorie und Theorie der Abwägung, Tubinga, Mohr Siebeck, pp. 155 y sigs.
} 
emergerían un amplio rango de soluciones. vez de enfocarme en las diferencias, me enfocaré en proponer un modelo basado en la teoría jurídica, un modelo que sirva como reconstrucción de un amplio denominador común en democracias liberales. Un instrumento clave en este modelo es la idea de principios jurídicos. Para explicar las características de los principios formales, primero necesita explicarse esta idea.

\section{La reconstrucción del principio de proporcionalidad por medio de la teoría de los principios}

\section{III.I. La teoría de los principios}

El fundamento de la teoría de los principios fue desarrollado por Ronald Dworkin en el debate sobre el positivismo jurídico en los años sesenta ${ }^{28}$, y esta teoría ha sido significativamente desarrollada con mayor atención por Robert Alexy desde la década de los ochenta en adelante ${ }^{29}$. En resumen, la distinción entre reglas y principios es la siguiente.

\section{A. Principios}

Los principios son normas que llevan a sí mismas a la ponderación. De hecho, cuando los principios entran en competencia y el conflicto no puede resolverse al apelar a otras medidas entonces requieren de ponderación. En otras palabras, los principios, a diferencia de las reglas, muestran la dimensión del peso ${ }^{30}$. Los principios requieren que una cierta meta sea optimizada y es por ello que han sido caracterizados como mandatos de optimización ${ }^{31}$. Cuando los principios entran en competencia, la ponderación determina qué principio tendrá precedencia en las circunstancias concretas del caso. No obstante, el principio derrotado continuará siendo válido $^{32}$. La ponderación es la forma característica de la aplicación de principios.

\section{B. Reglas}

Las reglas no llevan a la ponderación. Los conflictos de reglas llevan a la invalidez de una de las reglas mediante la introducción de una cláusula de excepción, siguiendo alguna de las máximas lex posterior derogat legi priori, lex specialis derogat, o legi generali lex superior derogat legi inferiori ${ }^{33}$. Esto quiere decir que los principios y reglas se diferencian por la dimensión del peso. Las exigencias provenientes de las reglas son juzgadas mediante la subsunción, la forma clásica de aplicación del derecho, en la cual los cánones de la interpretación, a saber, la interpretación

\footnotetext{
${ }^{28}$ DWORKIN (n. 14) $)$ pp. 22 y sigs.

${ }^{29}$ ALEXY, Robert (1985), "Rechtsregeln und Rechtsprinzipien”, en MACCORMICK, Neil et al, Geltungs- und Erkenntnisbedingungen im modernen Rechtsdenken, Stuttgart, Steiner, pp. 13-29; ALEXY (n. 9), pp. 44-69 (la edición original en alemán fue publicada por Suhrkamp en 1985).

${ }^{30}$ Ver DWORKIN, Ronald (n. 14), p. 26. El segundo criterio que Dworkin menciona, el llamado "todo o nada" ("all-or-nothing fashion”) (ibid., en p. 24), no sirve como criterio para distinguir entre reglas y principios. sobre esto, cfr., BOROWSKI, Martin (2010), “The Structure of Formal Principles”, en BOROWSKI, Martin (ed), On the Nature of Legal Principles, Stuttgart, Steiner, p. 22, con referencias posteriores.

${ }^{31}$ Ver ALEXY, Robert, (n. 9), pp. 47-8 y 67-69; ALEXY, Robert (200o), “On the Structure of Legal Principles”, Ratio Juris 13, p. 300.

${ }^{32}$ DWORKIN, Ronald (n. 16), p. 25; ALEXY, Robert (n. 9), p. 50.

${ }^{33}$ BOROWSKI, Martin (n. 32), p. 21 con referencias posteriores.
} 
literal, la intención histórica del legislador, la coherencia, el derecho como sistema y finalmente el propósito objetivo de la disposición legal, son claves.

\section{III.II. El análisis de la proporcionalidad y la teoría principialista}

Dworkin estaba lejos de la idea de conectar el principio de proporcionalidad en el derecho constitucional con los principios a ponderar, ya que la proporcionalidad no había sido claramente formulada y aceptada en el derecho constitucional de los Estados Unidos en ese tiempo ${ }^{34}$. No debería sorprendernos que esta conexión fuera hecha en Alemania, donde emergió la idea del principio de proporcionalidad. Con Robert Alexy, los principios jurídicos hicieron su camino del ámbito de la filosofía jurídica al derecho constitucional.

Para dar un ejemplo: el principio de protección al medio ambiente en la constitución alemana requiere que las decisiones de la autoridad pública sean ecológicamente viables. No obstante, es de sobra conocido que modos ecológicamente viables de producir bienes son con frecuencia más caros que modos de producir menos viables ecológicamente. Para buscar medios de producción ecológicamente viables se obliga al empresario, como regla, a escoger medios de producción más costosos. Esto interfiere con la libertad propia de conducir su libertad de empresa como quiera, libertad protegida como derecho constitucional bajo la constitución alemana. Desde el punto de vista de la teoría principialista, ambos derechos, el derecho individual -la libertad de conducir la libertad de empresa como se quiera- y el bien colectivo -la protección al medio ambiente- son principios, los cuales entran en colisión el uno con el otro. La ponderación de estos principios es llevada a cabo si una pretensión proveniente del derecho es considerada en el momento en el cual el análisis de proporcionalidad es realizado.

\section{Principios sustanciales y formales}

Una reconstrucción de la autoridad de las decisiones democráticas requiere de una distinción entre principios sustanciales y formales ${ }^{35}$. Esta reconstrucción será efectuada.

\section{IV.I. Principios sustanciales}

Los principios sustanciales son considerados razones para una decisión, razones que reflejan el contenido sustancial del principio ${ }^{36}$. Si volvemos al ejemplo mencionado antes, el principio de protección ambiental y la libertad de conducir la empresa como uno desee son principios sustanciales. Claro, uno puede argumentar acerca de qué principio sustancial tiene mayor peso que el otro según las circunstancias de un caso específico. Esto dependerá principalmente de tres factores ${ }^{37}$. El primero es el peso abstracto de los principios. Por ejemplo, la libertad de expresión es, desde un punto de vista abstracto, un principio de mayor peso, mientras que la libertad en general, libertad de hacer lo que no está específicamente tutelado en la constitución, es un principio con menor peso en la Ley Fundamental de Alemania. El segundo factor para asignar peso a un principio en un determinado caso es la intensidad de la interferencia o, en otras palabras, el grado de su no-realización. Mientras más severa sea la interferencia, más grande deberá ser el

\footnotetext{
${ }^{34}$ Sobre el debate respecto a si esto ha cambiado o debería cambiar, $c f r$., la nota 8.

${ }^{35}$ Sobre esta distinción, $c f r$., BOROWSKI, Martin (n. 30), pp. 24 y sigs; BOROWSKI, Martin (n. 27), pp. 184 y sigs

${ }^{36}$ DWORKIN, Ronald (n. 14), p. 38; BOROWSKI, Martin (n. 30), pp. 24-25; BOROWSKI, Martin (n. 27), pp. $185-186$.

${ }^{37}$ Sobre estos tres factores, $c f r$., ALEXY, Robert (n. 17), pp. 10 y sigs.; BOROWSKI, Martin (n. 10), pp. 82 y sigs. 
peso. Este segundo factor explica por qué no hay simplemente una jerarquía abstracta de valores o principios que decida los casos, ya que un principio con un alto peso abstracto puede ser superado por un principio con un peso abstracto menor, sólo si la interferencia con el principio posterior es más intensa que la interferencia con el primero. Esto dos factores han probado ser los más importantes. Existe, sin embargo, un tercer factor, la certeza epistémica, la cual tiene una función muy importante. Mientras más inciertas sean las premisas empíricas y normativas relevantes en la ponderación que será llevada a cabo, entonces se volverá más bajo el peso del principio en cuestión. Esto puede ser crucial para interferencias severas con principios con un alto peso abstracto, en las cuales no hay certeza de que, desde el punto de vista empírico, la interferencia realmente tendrá lugar.

La determinación de estas cantidades, a saber, peso abstracto, severidad de la interferencia y certeza de las premisas relevantes, resulta en argumentos normativos y empíricos. La justificación de estas cantidades no puede determinarse por el principio de proporcionalidad en tanto estructura. Esto quiere decir que el principio de proporcionalidad requiere ser complementado con una teoría de la argumentación jurídica ${ }^{38}$.

\section{IV.II. Principios formales}

Al ponderar principios sustanciales uno puede crear un sistema jurídico consistente y coherente. No obstante, es imposible describir adecuadamente la dimensión autoritativa del derecho al apelar sólo a principios sustanciales. A algunas decisiones se les atribuye peso no sólo por su contenido sustancial, sino porque representan un cierto procedimiento asumido por una autoridad jurídica.

\section{A. Principios formales en búsqueda de principios}

De hecho, los principios formales han sido introducidos en la búsqueda de principios para describir, en primer lugar, la dimensión autoritativa de ciertas decisiones jurídicas. Los comienzos de la oposición entre principios formales y sustanciales pueden encontrarse en Los derechos en serio de Dworkin. En sus consideraciones en torno a la discrecionalidad, Dworkin escribe: "[T]odo juez que se proponga cambiar la doctrina existente tiene que tomar en consideración algunos estándares importantes que son argumentados contra la doctrina establecida, y estos estándares son también parte de los principios"39. Los ejemplos de Dworkin incluyen a la "supremacía legislativa" un conjunto de principios que requieren que las cortes muestren un respeto a los actos legislativos" ${ }^{\circ}$. Tales principios, argumenta Dworkin, "se inclinan hacia el estatus quo" "1. Dworkin llama a estos principios "principios conservadores" y los contrasta con "principios sustanciales"42.

En su Teoría de los derechos fundamentales de 1985, Robert Alexy se refiere a una "distinción entre dos tipos de principios, que tiene consecuencias relevantes, es decir, la distinción en-

\footnotetext{
${ }^{38}$ BOROWSKI, Martin (n. 10), p. 121.

${ }^{39}$ DWORKIN, Ronald (n. 14), p. 37.

${ }^{40}$ Ibid.

${ }^{41}$ Ibid., pp. $37-38$

${ }^{42}$ Ibid., p. 38.
} 
tre principios sustanciales o materiales y principios formales o procedimentales" 43 El ejemplo principal de Alexy de un ejemplo procedimental es el "principio que establece que el legislador democrático debe tomar las decisiones importantes para la comunidad"44. Un par de páginas antes, después de analizar exposiciones sobre principios sustanciales, puede leerse lo siguiente: "[H]ay otros principios que también necesitan prevalecer, tal como aquel que establece que las normas aprobadas por una autoridad que actúa dentro de su jurisdicción deben seguirse, y el principio según el cual uno no debe apartarse de lo establecido sin una buena razón. Tales principios pueden llamarse 'principios formales"' 45.

\section{B. Críticas}

La idea de principios formales se ha enfrentado a diversas críticas. En síntesis, la objeción principal consiste en que los principios sustanciales y formales se encuentran en diferentes niveles, de modo tal que no pueden ponderarse los unos con los otros. También se ha sostenido que estas dos formas de principios son "inconmensurables” entre sí. De hecho, hasta ahora, la caracterización de los principios formales ha permanecido un tanto vaga y metafórica, y, por ello, se necesita un modelo más claro pare refutar estas objeciones.

\section{Un principio formal en la ponderación de principios sustanciales}

\section{V.I. La competencia para crear una meta a optimizar}

La clave para un entendimiento apropiado de los principios formales es que tal principio garantiza la competencia para crear una meta a optimizar en el sentido de la teoría principialista ${ }^{46}$. A diferencia de los principios sustantivos, los principios formales no manifiestan un contenido explícitamente establecido. Los principios formales se refieren un compromiso con los resultados de un procedimiento. Esto es, algo debe optimizarse, porque es resultado de cierto procedimiento o de la decisión de una autoridad. Una vez que el procedimiento ha sido llevado a cabo o la autoridad ha tomado una decisión, el contenido de la meta a fijar ha sido establecido. Ahora la estructura para ponderar un principio formal contra un principio sustantivo no difiere de ponderar dos principios sustantivos.

\section{V.II. La decisión autoritativa de ponderar principios sustanciales como la meta a optimizar del principio formal}

En el caso del principio formal que reconstruye la autoridad de una decisión del parlamento democráticamente legitimado, la decisión autoritativa es la decisión sobre la ponderación de principios sustantivos ${ }^{47}$. Esto quiere decir que este principio formal es dependiente de por lo menos dos principios sustanciales que necesitan ponderarse. La consideración del principio

\footnotetext{
${ }^{43}$ ALEXY, Robert (n. 9), p. 82.

${ }^{44}$ Ibid.

${ }^{45}$ Ibid., p. 58 (énfasis en el original).

${ }^{46}$ Sobre esta característica de los principios formales y sus repercusiones, cfr., BOROWSKI, Martin (n. 30), pp. 2930; BOROWSKI, Martin (n. 27), pp. 186-188.

${ }^{47}$ BOROWSKI, Martin (n. 30), p. 33; BOROWSKI, Martin (n. 27), pp. 190-194.
} 
formal tiene el efecto de agregar peso a uno de los dos principios sustantivos. A cuál principio se le agregará peso depende de la decisión del legislador parlamentario ${ }^{48}$.

\section{V.III. Tres escenarios}

Para ilustrar cómo un principio formal puede dar lugar a "discrecionalidad", pueden distinguirse tres escenarios. Estos escenarios serán expuestos a continuación.

Primer escenario: vamos a decir que, desde el punto de vista de la corte constitucional, al ponderar los principios sustanciales de protección al medio ambiente y la libertad de conducir el derecho de empresa de manera libre, el contenido máximo de dióxido de azufre en el gas residual es de 1100 ppm (partes por millón). Si suponemos que la ley parlamentaria prevé exactamente ese límite, el estatuto, obviamente, cumple con el estándar de control. La discreción no es un problema.

Segundo escenario: Supongamos que la ley parlamentaria, reconociendo una política particularmente amable con el medio ambiente, establece un límite inferior, $800 \mathrm{ppm}$ de $\mathrm{SO}_{2}$, mientras que el Tribunal Constitucional está convencido de que el límite debe ser de 1000 ppm de $\mathrm{SO}_{2}$. Este estatuto no es eo ipso inconstitucional. Por el contrario, éste demostrará ser constitucional si el principio sustantivo de la protección del medio ambiente tomado en conjunto con el principio formal que requiere prima facie que la decisión de ponderación del legislador parlamentario deba respetarse tiene mayor peso que el derecho para conducir la empresa propia como se desea como principio sustantivo en 800 ppm de $\mathrm{SO}_{2}$.

Tercer escenario: vamos a suponer que el estatuto parlamentario, reconociendo una política que favorece el crecimiento económico, establece un límite más alto, 1200 ppm de $\mathrm{SO}_{2}$, mientras que la corte constitucional está convencida de que tal derecho debe limitarse a $1000 \mathrm{ppm}$ de $\mathrm{SO}_{2}$. De nuevo, esta ley no es, eo ipso inconstitucional. El principio formal que requiere prima facie que la decisión a ponderar del legislador parlamentario sea respetada le agrega peso a la libertad de conducir el negocio propio como se quiera, de tal modo que estos dos principios tomados en conjunto pueden tener mayor peso que el principio sustantivo de protección al ambiente en $1200 \mathrm{ppm}$ de $\mathrm{SO}_{2}$ por metro cúbico de gas residual.

Ya sea que el principio formal le añada peso suficiente al principio sustantivo relevante de modo tal que ambos sobrepasen al principio sustantivo en competencia dependerá del peso de los dos principios sustanciales y del peso del principio formal en las circunstancias concretas. Mientras más peso se le atribuya al principio formal, más se alejará la decisión del legislador parlamentario de la mera ponderación de principios sustantivos. En síntesis: la mayor discreción es otorgada al legislador parlamentario ${ }^{49}$.

\section{V.IV. Los factores para otorgar peso al principio formal}

Ahora surge la pregunta en torno a qué factores determinan el peso del principio formal. La idea central es que estos factores se asemejan a los factores para determinar el peso de los principios sustantivos.

\footnotetext{
${ }^{48}$ BOROWSKI, Martin (n. 30), pp. 34-35; BOROWSKI, Martin (n. 27), pp. 194-195.

49 BOROWSKI, Martin (n. 30), pp. 34-35.
} 


\section{A. Peso abstracto}

Para empezar, un principio formal posee un peso abstracto ${ }^{50}$. Mientras más alto sea el peso abstracto, entonces mayor discrecionalidad será otorgada al parlamento legitimado democráticamente. Aquellos que simpatizan con el procedimiento democrático y con una aproximación procedimental argumentarán a favor de un peso abstracto elevado. Por el contrario, aquellos que enfaticen con el compromiso sustantivo del legislador de la constitución, establecida por una corte suprema o constitucional, argumentarán a favor de un peso abstracto bajo del principio formal.

\section{B. La intensidad de la interferencia}

Uno podría estar tentado a pensar que respecto a la decisión autoritativa nos encontramos ante un todo o nada (all-or-nothing-fashion), esto es, tú puedes respetar o no la decisión. No obstante, el respeto por una decisión autoritativa posee una dimensión de grado. Mientras más lejos se aparte uno de la decisión autoritativa, más grande se volverá el grado de no-realización, o, en otras palabras, más grande será la interferencia con el principio formal ${ }^{51}$. Si suponemos que el parlamento democráticamente legitimado decide que 800 ppm de dióxido de azufre es el límite y que la corte constitucional está convencida de ello, al ponderar la protección al medio ambiente y la libertad de manejar la empresa como se desee, de modo tal que la respuesta correcta debe ser 900 ppm de $\mathrm{SO}_{2}$, entonces la corte no está respetando la decisión del legislador del todo. Pero en esta situación la corte respeta más la decisión en este caso en comparación con un escenario en el cual la corte está convencida de que el resultado sólo puede ser de 3000 ppm de $\mathrm{SO}_{2}$. En el caso de 900 ppm de $\mathrm{SO}_{2}$, la corte sólo se aleja ligeramente de la decisión del parlamento democráticamente legitimado, mientras que en el caso de 3000 pm de $\mathrm{SO}_{2}$ se aleja significativamente. En el primer caso se trata de una interferencia menor con el principio formal, mientras que en el segundo caso se trata de una interferencia más intensa.

\section{Certeza epistémica}

Así como el peso de los principios sustantivos, el peso de los principios formales puede depender de la certeza epistémica de las premisas relevantes. Si uno plantea un paralelo con los principios sustantivos, la certeza epistémica, con el objeto de aplicarse al principio formal, sugiere mayor peso para este principio, mientras que la falta de certeza epistémica sugiere menor peso. Sin embargo, en el caso de principios formales, el aspecto en cuestión es la certeza de la decisión de la ponderación final y «certeza» se refiere tanto a la certeza epistémica-empírica como a la certeza epistémica-normativa ${ }^{52}$. En caso de que no haya falta de certeza epistémica del todo, el peso del principio formal desciende a cero ${ }^{53}$. Esto será para casos cuyas circunstancias prácticas, ya sean de falta de certeza normativa o empírica, sean excepciones muy raras ${ }^{54}$.

\footnotetext{
${ }^{50}$ BOROWSKI, Martin (n. 29), pp. 195-196.

${ }^{51}$ BOROWSKI, Martin (n. 29), pp. 196-197.

${ }^{52}$ BOROWSKI, Martin (n. 29), p. 197.

${ }^{53}$ ALEXY, Robert (n. 20), pp. 388-425, p. 424; ALEXY, Robert (2012), “Comments and Responses”, en KLATT, Matthias (ed), Institutionalized Reason. The Jurisprudence of Robert Alexy, Oxford, Oxford Uni-versity Press, p. 331; BOROWSKI (n. 27), p. 197.

54 BOROWSKI (n. 27), p. 197. 


\section{Características del peso de los principios formales y factores genéricos}

Aún si existen ciertos paralelos entre los factores que determinan el peso de principios formales y sustantivos, pueden existir también diferencias. Es decir, puede haber factores genéricos para el peso de los principios formales. Por ejemplo, de acuerdo con un famoso artículo de Peter Häberle de 1975, a la corte constitucional se le debe dar mayor discrecionalidad mientras más alta sea la calidad discursiva del proceso de legislación ${ }^{55}$. Si hubiera un debate profundo, justo y abierto, en el cual todos los argumentos relevantes fueran tomados en consideración y evaluados, esto contaría como un argumento para conseguir un menor escrutinio estricto o reducir el grado de discrecionalidad, en comparación con un proceso de legislación que ha sido fuertemente influido por grupos de presión ("lobbyists") y decidido básicamente a puertas cerradas y sin un debate democrático adecuado ${ }^{56}$. Si uno utiliza la teoría del discurso, tal y como ha sido desarrollada por Jürgen Habermas y Robert Alexy, la construcción resultante del control de constitucional en las democracias liberales iría en la misma dirección ${ }^{57}$.

\section{Conclusión}

A pesar de que todavía hay cuestiones que necesitarán atención antes de que lleguemos a un modelo detallado y completo para ponderar principios formales y materiales, los paralelos estructurales entre los principios sustanciales y formales, y los paralelos con la vista puesta en criterios para determinar su peso sugieren que de hecho ambos pueden considerarse en la misma ponderación. Las críticas son correctas al señalar que existen diferencias entre principios formales y sustanciales. No obstante, son incorrectas al sostener que estas diferencias se oponen a una consideración de los principios formales en la ponderación de principios sustanciales. Los principios formales y sustanciales difieren respecto a la conmensurabilidad en absoluto, pero esta diferencia es una cuestión de grado en vez de ser una diferencia categórica. Los principios formales y sustanciales operan de acuerdo con la misma lógica, y la consideración de ambos tipos de principios en la misma ponderación conecta o reconcilia la dimensión formal con la sustantiva, o, en otras palabras, corrección material con autoridad. Esto quiere decir que la objeción democrática al principio de proporcionalidad en el control de constitucionalidad puede ser refutada. La proporcionalidad en el control de constitucionalidad no puede violar los requisitos de la democracia, siempre y cuando estos requisitos sean considerados adecuadamente en los procesos de ponderación por medio de los principios formales.

\section{Bibliografía}

Alexy, Robert (1985), "Rechtsregeln und Rechtsprinzipien”, en: MACCORMICK, Neil et al, Geltungs- und Erkenntnisbedingungen im modernen Rechtsdenken, Stuttgart, Steiner: pp. 13-29.

\footnotetext{
${ }^{55}$ HÄBERLE, Peter (1975), "Die offene Gesellschaft der Verfassungsinterpreten”, Juristen Zeitung 10, pp. 297-305, pp. 303-304.

${ }^{56}$ BOROWSKI, Martin (2014), "Subjekte der Verfassungsinterpretation", en ISENSEE, Josef y KIRCHHOF, Paul (eds), Handbuch des Staatsrechts der Bundesrepublik Deutschland, vol. XII, Heidelberg, C.F. Müller, pp. 761-782, número marginal 42.

${ }^{57}$ Habría que agregar que HABERMAS, a diferencia de ALEXY, es bastante crítico respecto a la idea del control de constitucionalidad. sobre esto, $c f r$., n. 23 .
} 
Alexy, Robert (2000), “On the Structure of Legal Principles”, Ratio Juris 13: 294-304.

Alexy, Robert (2002), "Postscript", en: Robert Alexy, A Theory of Constitutional Rights (trad. De Julian Rivers), Oxford, Oxford University Press: pp. 388-425.

Alexy, Robert (2002), A Theory of Constitutional Rights (trad. de Julian Rivers), Oxford, Oxford University Press.

ALEXY, Robert (2003), "Die Gewichtsformel”, en JICKely, Joachim et al, Gedächtnisschrift für Jürgen Sonnenschein, Berlín, Duncker \& Humblot: pp. 771-792.

Alexy, Robert (2003), “On Balancing and Subsumption”, Ratio Juris 16: pp. 433-449.

Alexy, Robert (2007), “The Weight Formula”, en stelmach, Jerzy et al, Studies in the Philosophy of Law. Frontiers of the Economic Analysis of Law, Cracovia, Jagiellonian University Press: pp. 9-27.

Alexy, Robert (2012), “Comments and Responses”, en Klatt, Matthias (ed), Institutionalized Reason. The Jurisprudence of Robert Alexy, Oxford, Oxford University Press: pp. 319356.

Aleinikoff, Thomas Alexander (1986/1987), "Constitutional Law in the Age of Balancing", Yale Law Journal 96: pp. 943-1005.

BARAK, Aharon (2012), Proportionality, Cambridge, Cambridge University Press.

Beatty, David M. (2004), The Ultimate Rule of Law, Oxford, Oxford University Press.

Borowski, Martin (2009), "Die Bindung an Festsetzungen des Gesetzgebers in der grundrechtlichen Abwägung”, en: CLÉRICO, Laura y SIECKMANN, Jan-Reinard (eds), Grundrechte, Prinzipien und Argumentation, Baden-Baden, Nomos: pp. 99-128.

Bieber, Roland et al (2013), Die Europäische Union, décima edición, Baden-Baden, Nomos.

Borowski, Martin (2013), "Formelle Prinzipien und Gewichtsformel”, en: KLATT, Matthias (ed), Prinzipientheorie und Theorie der Abwägung, Tubinga, Mohr Siebeck: pp. 199-250.

Borowski, Martin (2007), Grundrechte als Prinzipien, segunda edición, Baden-Baden, Nomos.

Borowski, Martin (2012), "Libertades de Mercado y Derechos Fundamentales en la Unión Europea”, en CAPALDo, Griselda et al, Internacionalización del Derecho Constitucional, Constitucionalización del Derecho Internacional, Buenos Aires, Eudeba: pp. 383-398.

Borowski, Martin (2007), “Limiting Clauses”, en Legisprudence 1, pp. 197-240. 
Borowski, Martin (2010), “The Structure of Formal Principles”, en: M. Borowski (ed), On the Nature of Legal Principles, Stuttgart, Steiner: pp. 9-35.

BorowsKi, Martin (2014), "Subjekte der Verfassungsinterpretation", en ISENSEE, Josef y KIRCHHOF, Paul (eds), Handbuch des Staatsrechts der Bundesrepublik Deutschland, vol. XII, Heidelberg, C.F. Müller: pp. 761-782.

BÖCKENFÖRDE, Ernst-Wolfgang (1974), "Grundrechtstheorie und Grundrechtsinterpretation”, Neue juristische Wochenschrift 27: pp. 1529-1538.

BÖCKENFÖRDE, Ernst-Wolfgang (1992), “Grundrechte als Grundsatznormen”, en BÖCKENFÖRDE, Ernst-Wolfgang, Staat, Verfassung, Demokratie, segunda edición, Fráncfort del Meno, Suhrkamp: pp. 159-199.

BÖCKENFÖRDE, Ernst-Wolfgang (2003), "Schutzbereich, Eingriff, verfassungsimmanente Schranken. Zur Kritik gegenwärtiger Grundrechtsdogmatik”, Der Staat 42: pp. 165-192.

Cohen-Eliya, Moshe y Porat, Iddo (2010), “American Balancing and German Proportionality: The Historical Origins", International Journal of Constitutional Law 8: 263-286.

Craig, Paul y De Búrca, Gráinne (2011), EU Law - Text, Cases, and Materials, quinta edición, Oxford, Oxford University Press.

Dworkin, Ronald (1977), Taking Rights Seriously, Cambridge, Harvard University Press.

DüRIG, Günter (1956), "Der Grundrechtssatz von der Menschenwürde”, Archiv des öffentlichen Rechts 81: pp. 117-157.

Peters, Anne y Altwickler, Tilmann (2012), Europäische Menschenrechtskonvention, segunda edición, Múnich, C. H. Beck.

Harris, David et al (2009), Law of the European Convention on Human Rights, segunda edición, Oxford, Oxford University Press.

SCHLInk, Bernhard (1976), Abwägung im Verfassungsrecht, Berlín, Duncker \& Humblot.

Schlink, Bernhard (1992), "Grundrechte als Prinzipien?", Osaka University Law Review 39: 41-58.

Forsthoff, Ernst (1968), “Zur Situation einer heutigen Verfassungslehre“, en BARION, Hans et al, Epirrhosis. Festgabe für Carl Schmitt, Berlín, Duncker \& Humblot: pp. 185-211.

Frenz, Walter (2009), Handbuch Europarecht, Bd. 4 - Europäische Grundrechte, Berlín y Heidelberg, Springer.

Habermas, Jürgen (1996), Between Facts and Norms, trad. de William Rehg, Cambridge, Polity Press. 
Häberle, Peter (1975), “Die offene Gesellschaft der Verfassungsinterpreten”, Juristen Zeitung 10, pp. 297-305.

Jestaedt, Matthias (1999), Grundrechtsentfaltung im Gesetz, Tubinga, Mohr Siebeck.

KRÜGER, Herbert (1950), "Die Einschränkung von Grundrechten nach dem Grundgesetz", Deutsches Verwaltungsblatt 65: pp. 625-628.

LeISNER, Walter (1997), Der Abwägungsstaat, Berlín, Duncker \& Humblot.

Lerche, Peter (1961), Übermaß und Verfassungsrecht, Colonia, Carl Heymann.

Marauhn, Thilo y Merhof, Katrin (2013), “Grundrechtseingriff und -schranken”, en DörR, Oliver et al, EMRK/GG Konkordanzkommentar, vol. 1, Tubinga, Mohr Siebeck: pp. 366416.

MöLler, Kai (2007), "Balancing and the Structure of Constitutional Rights", International Constitutional Law 5: pp. 453-468.

Stern, Klaus (2011), “Idee und Elemente eines Systems der Grundrechte”, en ISENSEe, Josef y кIRснноғ, Paul (eds.), Handbuch des Staatsrechts der Bundesrepublik Deutschland, vol. IX, Heidelberg, C.F. Müller: pp. 57-120.

Sweet, Alec Stone y Matthews (2008), Jud, "Proportionality Balancing and Global Constitutionalism”, Columbia Journal of Transnational Law 47: pp. 73-165.

Tushnet, Mark (200o), Taking the Constitution away from the Courts, Princeton, Princeton University Press.

WALDRON, Jeremy (2006), “The Core of the Case against Judicial Review”, Yale Law Journal 115: pp. 1346-1406.

White, Robin y ovey, Clare (2010), The European Convention on Human Rights, quinta edición, Oxford, Oxford University Press. 